\title{
Strong and weak convergence of Mann iteration of mono- tone $\alpha$-nonexpansive mappings in uniformly convex Banach spaces
}

\author{
Yuchun Zheng ${ }^{\mathrm{a}, \mathrm{b}}$, Lin Wang ${ }^{\mathrm{a}, *}$ \\ ${ }^{a}$ College of Statistics and Mathematics, Yunnan University of Finance and Economics, Longquan Road, Kunming, 650221, P. R. China. \\ ${ }^{b}$ School of Mathematics and Information Science, Henan Normal University, XinXiang HeNan, 453007, P. R. China.
}

Communicated by Y. J. Cho

\begin{abstract}
In this paper, the demiclosed principle of monotone $\alpha$-nonexpansive mapping is showed in a uniformly convex Banach space with the partial order " $\leqslant$ ". With the help of such a demiclosed principle, the strong convergence of Mann iteration of monotone $\alpha$-nonexpansive mapping $\mathrm{T}$ are proved without some compact conditions such as semi-compactness of $\mathrm{T}$, and the weakly convergent conclusions of such an iteration are studied without the conditions such as Opial's condition. These convergent theorems are obtained under the iterative coefficient satisfying the condition,
\end{abstract}

$$
\sum_{k=1}^{+\infty} \min \left\{\alpha_{k},\left(1-\alpha_{k}\right)\right\}=+\infty,
$$

which contains $\alpha_{k}=\frac{1}{k+1}$ as a special case.

Keywords: Ordered Banach space, fixed point, monotone $\alpha$-nonexpansive mapping, strong convergence.

2010 MSC: 47H06, 47J05, 47J25, 47H10, 47H17, 49J40, 47H04, 65J15.

(C)2018 All rights reserved.

\section{Introduction}

In 1953, Mann [16] introduced the following iteration for finding a fixed point of a nonexpansive mapping $\mathrm{T}$,

$$
x_{k+1}=\alpha_{k} x_{n}+\left(1-\alpha_{k}\right) T x_{n} \text { for each positive integer } k,
$$

which was referred to as Mann iteration, where $\alpha_{k} \in[0,1]$ with some proper conditions. Subsequently, many mathematical workers paid their attentions to the strong and weak convergence of such an iteration

\footnotetext{
*Corresponding author

Email addresses: zhengyuchun@htu.cn (Yuchun Zheng), WL64mail@aliyun.com (Lin Wang)

doi: $10.22436 /$ jnsa.011.09.07
}

Received: 2017-05-30 Revised: 2018-03-22 Accepted: 2018-05-31 
and its modified version for many different mappings in the past several decades. For more details, see Opial [19] for nonexpansive mappings, Suzuki [27] for nonexpansive semigroups, Song [22] and Jung [10] for nonexpansive mappings sequence, Liu [15] for strongly accretive mappings, George and Nse [6] for hemi-contractive mappings, Kim et al. [11] for strictly hemi-contractive mappings, Okeke and Kim [18] for random Picard-Mann hybrid iterations, Berinde [3] and George and Shaini [7] for Zamfirescu operators, $\mathrm{Gu}$ and $\mathrm{Lu}$ [9] for nonlinear variational inclusions, and Zhou et al. [33], Song and Wang [25], Zhang and Su [31], and Zhou [32] for strict pseudo-contractions and the reference therein.

For a Banach space E endowed with the partial order " $\leqslant$ ", Bachar and Khamsi [2] introduced the concept of a monotone nonexpansive mapping. Let $T$ be a mapping with domain $D(T)$ and range $R(T)$ in $E$. $T: D(T) \rightarrow R(T)$ is said to be monotone nonexpansive if for all $x, y \in D(T)$ with $x \leqslant y$,

$$
T x \leqslant T y \text { and }\|T x-T y\| \leqslant\|x-y\| .
$$

Clearly, a monotone nonexpansive mapping may be discontinuous. Recently, Dehaish and Khamsi [4] showed weak convergence of the Mann iteration for monotone nonexpansive mappings in a uniformly convex Banach space with Opial's condition. Narghirad et al. [17] studied iteration approximation and fixed point theory for $\alpha$-nonexpansive mappings in CAT(0) spaces. Very recently, Song et al. [24] first discussed the properties of monotone $\alpha$-nonexpansive mappings. $T: D(T) \rightarrow R(T)$ is said to be monotone $\alpha$-nonexpansive if for all $x, y \in D(T)$ with $x \leqslant y$,

$$
\mathrm{T} x \leqslant \mathrm{~T} y \text { and }\|\mathrm{T} x-\mathrm{T} y\|^{2} \leqslant \alpha\|\mathrm{T} x-y\|^{2}+\alpha\|x-\mathrm{T} y\|^{2}+(1-2 \alpha)\|x-y\|^{2} .
$$

Still in a uniformly convex Banach space with Opial's condition, Song et. al. [24] showed weak convergence of Mann iteration for monotone $\alpha$-nonexpansive mappings under the iteration coefficient satisfying the conditions,

$$
\limsup _{k \rightarrow \infty} \alpha_{k}\left(1-\alpha_{k}\right)>0 \text { or } \liminf _{k \rightarrow \infty} \alpha_{k}\left(1-\alpha_{k}\right)>0,
$$

which excludes $\alpha_{k}=0$ or $\frac{1}{k+1}$ for all positive integers $k$.

It is well-known that the Mann iteration is called Picard iteration if $\alpha_{k}=0$ in Mann iteration (1.1). In 1971, Pazy [20] proved the equivalence relation between strong convergence of Picard iteration and the existence of fixed point for nonexpansive mappings in Hilbert spaces, which is referred to as Pazy's Fixed Point Theorem. Under the frame of Hilbert space, strong convergences of Picard iteration were showed by Kohsaka and Takahashi [13] for a nonspreading mapping, and by Takahashi [28] for a hybrid mapping, and Takahashi and Yao [30] for a TJ-mapping, and Lin and Wang [14] for an (a,b)-monotone mapping. In a uniformly convex Banach space with the partial order " $\leqslant$ ", Pazy's Fixed Point Theorem was proved by Song et al. [23] for a monotone nonexpansive mapping, and by Song and Chen [21] for a monotone $\alpha$-nonexpansive mapping. Naturally, we may have the following question:

May the natural sequence $\left\{\frac{1}{\mathrm{k}+1}\right\}$ ensure strong or weak convergence of Mann iteration for a monotone $\alpha$-nonexpansive mapping?

In this paper, we give an affirmative answer in a uniformly convex Banach space with the partial order " $\leqslant$ " with respective to a normal cone P. More precisely, the weakly and strongly convergent conclusions of Mann iteration are showed for a monotone $\alpha$-nonexpansive mapping under the iterative coefficient satisfying the condition,

$$
\sum_{k=1}^{+\infty} \min \left\{\alpha_{k},\left(1-\alpha_{k}\right)\right\}=+\infty .
$$

Clearly, the above condition contains $\alpha_{k}=\frac{1}{k+1}$ as a special case. In particular, the weak convergence of Mann iteration does not require that Banach space satisfies Opial's condition, and the strong convergence of such an iteration is proved for monotone $\alpha$-nonexpansive mapping $T$ without some compact conditions such as semi-compact of T. Furthermore, we also obtain the demiclosed principle of monotone $\alpha$-nonexpansive mapping $T$ in a uniformly convex Banach space $E$ with the partial order " $\leqslant$. 


\section{Preliminaries and basic results}

Throughout this paper, let $E$ be a real Banach space with the norm $\|\cdot\|$ and the partial order " $\leqslant$ ". Let $F(T)=\{x \in E: T x=x\}$ stand for the set of all fixed points of a mapping $T$.

Let $\mathrm{P}$ be a nonempty, closed, convex cone of $\mathrm{E}$. We define the partial order " $\leqslant$ " with respect to $\mathrm{P}$ as follows: for all $x, y \in E$,

$$
x \leqslant y \text { if and only if } y-x \in P .
$$

The partial order " $\geqslant$ " is given by $x \geqslant y$ if and on if $y \leqslant x$. Then it is obvious that

$$
x=y \text { if and on if } x \leqslant y \text { and } x \geqslant y .
$$

Clearly, for all $t$ with $0 \leqslant t \leqslant 1$ and all $x, y \in E$, the fact that $x \leqslant y$ means that

$$
x \leqslant t x+(1-t) y \leqslant y .
$$

Lemma 2.1 ([26]). Let $\mathrm{E}$ be a Banach space with the partial order " $\leqslant$ ". Assume that $\left\{x_{\mathrm{k}}\right\}$ and $\left\{\mathrm{y}_{\mathrm{k}}\right\}$ are two sequences on $\mathrm{E}$ such that

$$
x_{k} \leqslant y_{k} \text { for each positive integer } k .
$$

If $\left\{x_{k}\right\}$ and $\left\{y_{k}\right\}$ weakly converge to $x$ and $y$, respectively, then

$$
x \leqslant y .
$$

Definition $2.2([2,24])$. Let $K$ be a nonempty closed convex subset of an ordered Banach space $(E, \leqslant)$. A mapping $\mathrm{T}: \mathrm{K} \rightarrow \mathrm{E}$ is said to be:

(1) monotone ([2]) if $\mathrm{Tx} \leqslant \mathrm{Ty}$ for all $x, y \in \mathrm{K}$ with $x \leqslant y$;

(2) monotone nonexpansive ([2]) if $\mathrm{T}$ is monotone and

$$
\|T x-T y\| \leqslant\|x-y\|
$$

for all $x, y \in K$ with $x \leqslant y$.

(3) monotone $\alpha$-nonexpansive ([24]) if $\mathrm{T}$ is monotone and for some $\alpha<1$,

$$
\|\mathrm{T} x-\mathrm{T} y\|^{2} \leqslant \alpha\|\mathrm{T} x-y\|^{2}+\alpha\|\mathrm{T} y-x\|^{2}+(1-2 \alpha)\|x-y\|^{2}
$$

for all $x, y \in K$ with $x \leqslant y$;

(4) monotone quasi-nonexpansive ([24]) if $\mathrm{F}(\mathrm{T}) \neq \emptyset$ and

$$
\|T x-p\| \leqslant\|x-p\|
$$

for all $p \in F(T)$ and all $x \in K$ with $x \leqslant p$ or $x \geqslant p$.

Lemma 2.3 ([24, Lemma 2.1]). Let $\mathrm{K}$ be a nonempty closed convex subset of an ordered Banach space $(\mathrm{E}, \leqslant)$ and $\mathrm{T}: \mathrm{K} \rightarrow \mathrm{K}$ be a monotone $\alpha$-nonexpansive mapping. Then

(1) $\mathrm{T}$ is monotone quasi-nonexpansive;

(2) for all $x, y \in K$ with $x \leqslant y$ (or $y \leqslant x)$,

$$
\|T x-T y\|^{2} \leqslant\|x-y\|^{2}+\frac{2 \alpha}{1-\alpha}\|T x-x\|^{2}+\frac{2|\alpha|}{1-\alpha}\|T x-x\|(\|x-y\|+\|T x-T y\|) .
$$

Definition 2.4. Let $E$ ba a Banach space. 
(i) A function $\delta_{E}:[0,2] \rightarrow[0,1]$ is said to be the modulus of convexity of $E$ if

$$
\delta_{\mathrm{E}}(\varepsilon)=\inf \left\{1-\frac{\|x+y\|}{2} ;\|x\| \leqslant 1,\|y\| \leqslant 1,\|x-y\| \geqslant \varepsilon\right\} ;
$$

(ii) $\mathrm{E}$ is called uniformly convex if $\delta_{\mathrm{E}}(\varepsilon)>0$ for all $\varepsilon \in(0,2]$.

The following properties of the modulus of convexity of a Banach space $E$ may be found in the references $[1,8,12]$.

Lemma 2.5 ([1, Corollary 2.3.11]). Let $\mathrm{E}$ be a uniformly convex Banach space with the modulus of convexity $\delta_{\mathrm{E}}(\cdot)$. Then for $r>0$ and $x, y \in E$ with $\|x\| \leqslant r,\|y\| \leqslant r$,

$$
\|\lambda x+(1-\lambda) y\| \leqslant r\left[1-2 \min \{\lambda, 1-\lambda\} \delta_{\mathrm{E}}\left(\frac{\|x-y\|}{r}\right)\right] \text { for all } \lambda \in(0,1) .
$$

In particular, taking $\lambda=\frac{1}{2}$,

$$
\left\|\frac{x+y}{2}\right\| \leqslant r\left[1-\delta_{E}\left(\frac{\|x-y\|}{r}\right)\right] .
$$

The following conclusion is well known. For the more detail, see the references $[5,12,29]$.

Lemma 2.6. Let $\mathrm{C}$ be a nonempty closed convex subset of a reflexive Banach space $\mathrm{E}$. Assume that $\mathrm{f}: \mathrm{C} \rightarrow$ $(-\infty,+\infty)$ is a proper convex lower semi-continuous and coercive function (i.e., $\left.\lim _{\|x\| \rightarrow \infty} f(x)=\infty\right)$. Then there exists $x \in \mathrm{C}$ such that

$$
f(x)=\inf _{y \in C} f(y)
$$

\section{Main results}

In this section, we first show the demi-closed principle for monotone $\alpha$-nonexpansive mapping.

Theorem 3.1. Let $\mathrm{K}$ be a nonempty and closed convex subset of a uniformly convex Banach space $\mathrm{E}$ with the partial order " $\leqslant$ ", and let $\mathrm{T}: \mathrm{K} \rightarrow \mathrm{K}$ be a monotone $\alpha$-nonexpansive mapping. Assume that the norm $\|\cdot\|$ is monotonic, i.e.,

$$
\|x\| \leqslant\|y\| \text { for all } x, y \in E \text { with } 0 \leqslant x \leqslant y .
$$

If a sequence $\left\{x_{k}\right\} \subset K$ weakly converges to $x$ with $x_{k} \leqslant T x_{k} \leqslant x\left(\right.$ or $\left.x_{k} \geqslant T x_{k} \geqslant x\right)$ and

$$
\lim _{k \rightarrow \infty}\left\|x_{k}-T x_{k}\right\|=0
$$

then $x=T x$.

Proof. Without loss of generality, we may assume that $x_{k} \leqslant T x_{k} \leqslant x$ for all positive integers $k$. Let $C=\left\{y \in K ; x_{k} \leqslant y\right.$ for all positive integers $\left.k\right\}$. Then, $C=\bigcap_{k=1}^{\infty} C_{k}$, where $C_{k}=\left\{y \in K ; x_{k} \leqslant y\right\}$. Clearly, $C_{k}$ is nonempty, closed convex for all positive integers $k$, and hence, $C \subset K$ is nonempty closed convex $(x \in C)$. It follows from the weak convergence of $\left\{x_{k}\right\}$ that $\left\{x_{k}\right\}$ is bounded, which together with the fact that $\lim _{k \rightarrow \infty}\left\|x_{k}-T x_{k}\right\|=0$ implies the boundedness of $\left\{T x_{k}\right\}$. So, we may define a function $\varphi: C \rightarrow[0,+\infty)$ as follows

$$
\varphi(y)=\limsup _{k \rightarrow \infty}\left\|x_{k}-y\right\| \text { for all } y \in C .
$$

It is obvious that $\varphi$ is a proper convex, continuous, and coercive function. It follows from Lemma 2.6 that there exists $z \in C$ such that

$$
\varphi(z)=\limsup _{k \rightarrow \infty}\left\|x_{k}-z\right\|=\inf _{y \in C} \varphi(y)=r,
$$


and by the definition of $C$, we have

$$
x_{k} \leqslant z \text { for all positive integers } k \text {. }
$$

From Lemma 2.1, it follows that $x_{k} \leqslant x \leqslant z$, and hence,

$$
0 \leqslant x-x_{k} \leqslant z-x_{k} \text { for all positive integers } k \text {, }
$$

which implies that

$$
\left\|x-x_{k}\right\| \leqslant\left\|z-x_{k}\right\| \text { and so, } \varphi(x) \leqslant \varphi(z) .
$$

Therefore, we have

$$
\varphi(x)=\varphi(z)=\limsup _{k \rightarrow \infty}\left\|x_{k}-x\right\|=\inf _{y \in C} \varphi(y)=r .
$$

By the monotonicity of $T$, we have also, $x_{k} \leqslant T x_{k} \leqslant T x$, and hence, $T x \in C$. Thus $\frac{x+T x}{2} \in C$ by the convexity of $\mathrm{C}$, and so,

$$
r=\varphi(x) \leqslant \varphi\left(\frac{x+T x}{2}\right) \text { and } r=\varphi(x) \leqslant \varphi(T x) .
$$

From Lemma 2.3, it follows that

$$
\left\|T x_{k}-T x\right\|^{2} \leqslant\left\|x_{k}-x\right\|^{2}+\frac{2 \alpha}{1-\alpha}\left\|T x_{k}-x_{k}\right\|^{2}+\frac{2|\alpha|}{1-\alpha}\left\|T x_{k}-x_{k}\right\|\left(\left\|x_{k}-x\right\|+\left\|T x_{k}-T x\right\|\right),
$$

and hence, we have $\left(\limsup _{k \rightarrow \infty}\left\|T x_{k}-T x\right\|\right)^{2} \leqslant\left(\limsup _{k \rightarrow \infty}\left\|x_{k}-x\right\|\right)^{2}$, i.e.,

$$
\limsup _{k \rightarrow \infty}\left\|T x_{k}-T x\right\| \leqslant \limsup _{k \rightarrow \infty}\left\|x_{k}-x\right\|=\varphi(x) .
$$

Thus, using the inequality $\left\|x_{k}-T x\right\| \leqslant\left\|x_{k}-T x_{k}\right\|+\left\|T x_{k}-T x\right\|$, we obtain that

$$
\varphi(T x)=\limsup _{k \rightarrow \infty}\left\|x_{k}-T x\right\| \leqslant \limsup _{k \rightarrow \infty}\left\|T x_{k}-T x\right\| \leqslant \limsup _{k \rightarrow \infty}\left\|x_{k}-x\right\|=\varphi(x)=r .
$$

So, using the inequality $\left\|x_{k}-\frac{x+T x}{2}\right\| \leqslant \frac{1}{2}\left\|x_{k}-x\right\|+\frac{1}{2}\left\|x_{k}-T x\right\|$, we also have

$$
\varphi\left(\frac{x+T x}{2}\right) \leqslant \frac{1}{2} \limsup _{k \rightarrow \infty}\left\|x_{k}-x\right\|+\frac{1}{2} \limsup _{k \rightarrow \infty}\left\|x_{k}-T x\right\| \leqslant \limsup _{k \rightarrow \infty}\left\|x_{k}-x\right\|=\varphi(x)=r .
$$

Combining (3.1), (3.2), and (3.3), we get

$$
\varphi(x)=\varphi(T x)=\varphi\left(\frac{x+T x}{2}\right)=r \geqslant 0 .
$$

Now we show $x=T x$. In fact, suppose $r=0$. Then $\lim _{k \rightarrow \infty}\left\|x_{k}-T x\right\|=\lim _{k \rightarrow \infty}\left\|x_{k}-x\right\|=0$, which implies $x=T x$. If $r>0$, then from the definition of the upper limit "lim sup", it follows that for all $\varepsilon>0$, there is a positive integer $l$ such that

$$
\left\|x_{k}-T x\right\|<r+\varepsilon \text { and }\left\|x_{k}-x\right\|<r+\varepsilon \text { for all positive integers } k>l .
$$

So by Lemma 2.5 , we have

$$
\left\|x_{k}-\frac{x+T x}{2}\right\|=\left\|\frac{1}{2}\left(x_{k}-x\right)+\frac{1}{2}\left(x_{k}-T x\right)\right\| \leqslant(r+\varepsilon)\left(1-\delta_{E}\left(\frac{\|x-T x\|}{r+\varepsilon}\right)\right) .
$$


We may restrict that $\varepsilon<1$ without loss of generality. Then the above inequality can be rewritten as follow:

$$
\left\|x_{k}-\frac{x+T x}{2}\right\| \leqslant(r+\varepsilon)\left(1-\delta_{E}\left(\frac{\|x-T x\|}{r+1}\right)\right)
$$

and hence,

$$
r=\varphi\left(\frac{x+T x}{2}\right)=\limsup _{k \rightarrow \infty}\left\|x_{k}-\frac{x+T x}{2}\right\| \leqslant(r+\varepsilon)\left(1-\delta_{E}\left(\frac{\|x-T x\|}{r+1}\right)\right) .
$$

Therefore, we have

$$
r \delta_{E}\left(\frac{\|x-T x\|}{r+1}\right) \leqslant(r+\varepsilon) \delta_{E}\left(\frac{\|x-T x\|}{r+1}\right) \leqslant r+\varepsilon-r=\varepsilon .
$$

Since $\varepsilon$ is arbitrary, we have

$$
\delta_{E}\left(\frac{\|x-T x\|}{r+1}\right)=0
$$

and hence, $x=T x$. The desired conclusion follows.

When $x_{k} \geqslant T x_{k} \geqslant x$ for all positive integers $k$, we only need set

$$
C=\left\{y \in K ; x_{k} \geqslant y \text { for all positive integers } k\right\} .
$$

The remainder of proof is same, so we omit it.

In the sequel, we will use the fixed point set with the partial orders $F_{\leqslant}^{x}(T)$ and $F_{\geqslant}^{x}(T)$ given by

$$
F_{\leqslant}^{x}(T)=\{p \in F(T): p \leqslant x\} \text { for some } x \in K
$$

and

$$
F_{\geqslant}^{x}(T)=\{p \in F(T): p \geqslant x\} \text { for some } x \in K,
$$

respectively. Next we study the convergence of the Mann iteration of a monotone $\alpha$-nonexpansive mapping $T$ in an ordered uniformly convex Banach space $(E, \leqslant)$ by means of the proof technique of Song and Chen [21]. For an initial point $x_{1} \in K \subset E$, the sequence $\left\{x_{n}\right\}$ given by the Mann iteration is

$$
x_{k+1}=\alpha_{k} x_{k}+\left(1-\alpha_{k}\right) T x_{k} .
$$

Theorem 3.2. Let $\mathrm{K}$ be a nonempty, closed convex subset of a uniformly convex Banach space $\mathrm{E}$ with a partial order " $\leqslant$ ", and let $\mathrm{T}: \mathrm{K} \rightarrow \mathrm{K}$ be a monotone $\alpha$-nonexpansive mapping. Assume that the norm $\|\cdot\|$ is monotonic and the sequence $\left\{x_{k}\right\}$ defined by the Mann iteration (3.4) with $\mathrm{x}_{1} \leqslant \mathrm{~T} \mathrm{x}_{1}$ and $\mathrm{F}_{\geqslant}^{\mathrm{x}_{1}}(\mathrm{~T}) \neq \emptyset$. If the iteration coefficients $\left\{\alpha_{k}\right\} \subset(0,1)$ satisfy

$$
\sum_{k=1}^{+\infty} \gamma_{k}=+\infty, \gamma_{k}=\min \left\{\alpha_{k},\left(1-\alpha_{k}\right)\right\} \text { for all positive integers } k
$$

then $\left\{x_{k}\right\}$ weakly converges to some fixed point $x \in \mathrm{F}_{\geqslant}^{x_{1}}(T)$ and $x \geqslant x_{k}$ for all $k$.

Proof. Choose $p \in \mathrm{F}_{\geqslant}^{x_{1}}(T)$. We show by mathematical induction that $p \geqslant x_{k+1} \geqslant x_{k}$ for all positive integers $k$. From the monotonicity of $T$ along with the fact that $x_{1} \leqslant p$, it follows that

$$
x_{1} \leqslant T x_{1} \leqslant T p=p,
$$

and so, by the definition of Mann iteration (3.4) $\left(x_{2}=\gamma_{1} x_{1}+\left(1-\alpha_{1}\right) T x_{1}\right)$, we have

$$
x_{1} \leqslant x_{2} \leqslant T x_{1} \leqslant p .
$$


Suppose that $x_{k} \leqslant p$. Then $T x_{k} \leqslant T p=p$, and hence, from the definition of Mann iteration (3.4), it follows that

$$
x_{k} \leqslant x_{k+1} \leqslant T x_{k} \leqslant p,
$$

as claimed. That is, we obtain that the sequence $\left\{x_{k}\right\}$ is increasing with respect to the partial order " $\leqslant$ " and $p$ is a upper bound with respect to the partial order " $\leqslant$ ".

It follows from Lemma 2.3 that

$$
\left\|T x_{k}-p\right\| \leqslant\left\|x_{k}-p\right\| \text { for all positive integers } k,
$$

and hence, we have

$$
\begin{aligned}
\left\|x_{k+1}-p\right\| & \leqslant \alpha_{k}\left\|x_{k}-p\right\|+\left(1-\alpha_{n}\right)\left\|T x_{k}-p\right\| \\
& \leqslant \alpha_{k}\left\|x_{k}-p\right\|+\left(1-\alpha_{k}\right)\left\|x_{k}-p\right\|=\left\|x_{k}-p\right\| \leqslant \cdots \leqslant\left\|x_{1}-p\right\| .
\end{aligned}
$$

Then the sequence $\left\{\left\|x_{k}-p\right\|\right\}$ is nonincreasing and bounded for all $p \in F_{\geqslant}^{x_{1}}(T)$, and so, the $\operatorname{limit}_{k \rightarrow \infty} \| x_{k}-$ $p \|$ exists. Furthermore, both sequences $\left\{x_{k}\right\}$ and $\left\{T x_{k}\right\}$ are bounded with respect to the norm " $\|\cdot\|$ ". Since $E$ is uniformly convex, the boundedness of $\left\{x_{k}\right\}$ implies that $\left\{x_{k}\right\}$ is weakly sequentially compact. Then there exists a subsequence $\left\{x_{k_{l}}\right\}$ such that $\left\{x_{k_{l}}\right\}$ weakly converge to some point $x$. For each fixed $k$, there exists large enough $k_{l}$ such that $x_{k} \leqslant x_{k_{l}}$. From Lemma 2.1, it follows that $x_{k} \leqslant x$. Since $k$ is arbitrary, $x_{k} \leqslant x$ for all positive integers $k$.

We claim that $\left\{x_{k}\right\}$ weakly converges to $x$. Suppose not. Then there is a subsequence $\left\{x_{k_{j}}\right\}$ of $\left\{x_{k}\right\}$ such that $\left\{x_{k_{j}}\right\}$ weakly converge to $y \neq x$. For each fixed $k_{l}$, there is large enough $k_{j}$ such that $x_{k_{l}} \leqslant x_{k_{j}}$. Then by Lemma 2.1, we have $x_{k_{l}} \leqslant y$. Since $\left\{x_{k_{l}}\right\}$ weakly converges to $x$, then $x \leqslant y$. Using the same proof technique, we also have $y \leqslant x$. Thus $x=y$, which is a contradiction. So the claim is proved.

Now we show $x \in F_{\geqslant}^{x_{1}}(T)$. In fact, suppose $\lim _{k \rightarrow \infty}\left\|x_{k}-p\right\|=0$. Then we must have $x=p$ since $\left\{x_{k}\right\}$ weakly converges to $x$. Next we assume that

$$
\lim _{k \rightarrow \infty}\left\|x_{k}-p\right\|=t>0
$$

Then there exist two positive numbers $a, b$ and some positive integer $\mathrm{N}$ such that

$$
0<a \leqslant\left\|x_{k}-p\right\| \leqslant b \text { for all } k>N .
$$

On the other hand, it follows from Lemma $2.5\left(r=\left\|x_{k}-p\right\|\right.$ and $\lambda=\alpha_{k}$ for each $\left.k>N\right)$ that for each positive integer $\mathrm{k}>\mathrm{N}$,

$$
\begin{aligned}
\left\|x_{k+1}-p\right\| & =\left\|\alpha_{k}\left(x_{k}-p\right)+\left(1-\alpha_{k}\right)\left(T x_{k}-p\right)\right\| \\
& \leqslant\left\|x_{k}-p\right\|\left(1-2 \min \left\{\alpha_{k}, 1-\alpha_{k}\right\} \delta_{E}\left(\frac{\left\|x_{k}-T x_{k}\right\|}{\left\|x_{k}-p\right\|}\right)\right) \\
& \leqslant\left\|x_{k}-p\right\|\left(1-2 \gamma_{k} \delta_{E}\left(\frac{\left\|x_{k}-T x_{k}\right\|}{b}\right)\right),
\end{aligned}
$$

which implies that

$$
a \gamma_{k} \delta_{E}\left(\frac{\left\|x_{k}-T x_{k}\right\|}{b}\right) \leqslant 2\left\|x_{k}-p\right\| \gamma_{k} \delta_{E}\left(\frac{\left\|x_{k}-T x_{k}\right\|}{b}\right) \leqslant\left\|x_{k}-p\right\|-\left\|x_{k+1}-p\right\| .
$$

Then, we have

$$
\sum_{k=N+1}^{n} a \gamma_{k} \delta_{E}\left(\frac{\left\|x_{k}-T x_{k}\right\|}{b}\right) \leqslant \sum_{k=N+1}^{n}\left(\left\|x_{k}-p\right\|-\left\|x_{k+1}-p\right\|\right)=\left\|x_{N+1}-p\right\|-\left\|x_{n}-p\right\|,
$$


and hence,

$$
\sum_{k=N+1}^{+\infty} a \gamma_{k} \delta_{E}\left(\frac{\left\|x_{k}-T x_{k}\right\|}{b}\right) \leqslant \lim _{n \rightarrow+\infty}\left(\left\|x_{N+1}-p\right\|-\left\|x_{n}-p\right\|\right)<+\infty .
$$

Therefore, we must have

$$
\liminf _{k \rightarrow \infty} \delta_{E}\left(\frac{\left\|x_{k}-T x_{k}\right\|}{b}\right)=0 .
$$

Suppose not, $\liminf _{k \rightarrow \infty} \delta_{E}\left(\frac{\left\|x_{k}-T x_{k}\right\|}{b}\right)>0$. Then there exists $s>0$ and some positive integer $n$ such that

$$
\delta_{E}\left(\frac{\left\|x_{k}-T x_{k}\right\|}{b}\right) \geqslant s>0 \text { for all } k>n,
$$

and so, we have

$$
a \gamma_{k} \delta_{E}\left(\frac{\left\|x_{k}-T x_{k}\right\|}{b}\right) \geqslant a s \gamma_{k}
$$

By the condition that $\sum_{k=1}^{+\infty} a \gamma_{k}=+\infty$, we have $\sum_{k=1}^{+\infty} \gamma_{k} \delta_{E}\left(\frac{\left\|x_{k}-T x_{k}\right\|}{b}\right)=+\infty$, which is a contradiction. So, the equation (3.5) holds. It follows from the properties of the modulus of convexity $\delta_{\mathrm{E}}(\cdot)$ that

$$
\liminf _{k \rightarrow \infty}\left\|x_{k}-T x_{k}\right\|=0
$$

and so, there exists a subsequence $\left\{x_{k_{i}}\right\}$ of $\left\{x_{k}\right\}$ such that $\lim _{i \rightarrow \infty}\left\|x_{k_{i}}-T x_{k_{i}}\right\|=0$. It is obvious that $\left\{x_{k_{i}}\right\}$ weakly converges to $x$ since $\left\{x_{k}\right\}$ weakly converges to $x$. Thus, from Theorem 3.1, it follows that $x=T x$, i.e., $x \in \mathrm{F}_{\geqslant}^{\mathrm{x}_{1}}(\mathrm{~T})$. The desired conclusion follows.

Using the same proof technique, we easily obtain the following theorem.

Theorem 3.3. Let $\mathrm{K}$ be a nonempty, closed convex subset of a uniformly convex Banach space $\mathrm{E}$ with a partial order " $\leqslant$, and let $\mathrm{T}: \mathrm{K} \rightarrow \mathrm{K}$ be a monotone $\alpha$-nonexpansive mapping. Assume that the norm $\|\cdot\|$ is monotonic and the sequence $\left\{x_{k}\right\}$ defined by the Mann iteration (3.4) with $\mathrm{T}_{1} \leqslant \mathrm{x}_{1}$ and $\mathrm{F}_{\leqslant}^{\mathrm{x}_{1}}(\mathrm{~T}) \neq \emptyset$. If the iteration coefficients $\left\{\alpha_{\mathrm{k}}\right\}$ satisfy

$$
\sum_{k=1}^{+\infty} \gamma_{k}=+\infty, \gamma_{k}=\min \left\{\alpha_{k},\left(1-\alpha_{k}\right)\right\} \text { for all positive integers } k,
$$

then $\left\{x_{k}\right\}$ weakly converges to some fixed point $x \in \mathrm{F}_{\leqslant}^{x_{1}}(T)$ and $x \leqslant x_{k}$ for all $k$.

It is well known that the cone $P$ is normal if and only if there exists a equivalent norm $\|\cdot\|_{1}$ which is monotonic. So the following corollary is obvious.

Corollary 3.4. Let $\mathrm{E}$ be a uniformly convex Banach space with the partial order " $\leqslant$ " with respect to the normal cone $\mathrm{P}$, and let $\mathrm{K}$ be a nonempty closed convex subset of $\mathrm{E}$, and let $\mathrm{T}: \mathrm{K} \rightarrow \mathrm{K}$ be a monotone $\alpha$-nonexpansive mapping. Assume that the sequence $\left\{x_{\mathrm{k}}\right\}$ defined by the Mann iteration (3.4) with $\mathrm{T} \mathrm{x}_{1} \leqslant \mathrm{x}_{1}$ and $\mathrm{F}_{\leqslant}^{\mathrm{x}_{1}}(\mathrm{~T}) \neq \emptyset$ (or $\mathrm{T} \mathrm{x}_{1} \geqslant \mathrm{x}_{1}$ and $\mathrm{F}_{\geqslant} \mathrm{x}_{1}(\mathrm{~T}) \neq \emptyset$ ). If the iteration coefficients $\left\{\alpha_{\mathrm{k}}\right\}$ satisfy

$$
\sum_{k=1}^{+\infty} \gamma_{k}=+\infty, \gamma_{k}=\min \left\{\alpha_{k},\left(1-\alpha_{k}\right)\right\} \text { for all positive integers } k,
$$

then $\left\{x_{k}\right\}$ weakly converges to some fixed point $x \in \mathrm{F}(\mathrm{T})$.

Let the intersection of the domain $\mathrm{D}(\mathrm{T})$ of monotone $\alpha$-nonexpansive mapping $\mathrm{T}$ and the cone $\mathrm{P}$ is nonempty, i.e., $\mathrm{D}(\mathrm{T}) \cap \mathrm{P} \neq \emptyset$. The following strongly convergent theorems may be obtained by means of the proof technique of Song and Chen [21]. 
Theorem 3.5. Let $\mathrm{K}$ be a nonempty, closed convex subset of a uniformly convex Banach space $\mathrm{E}$ with a partial order " $\leqslant$ ", and let $\mathrm{T}: \mathrm{K} \rightarrow \mathrm{K}$ be a monotone $\alpha$-nonexpansive mapping. Assume that the norm $\|\cdot\|$ is monotonic and the sequence $\left\{x_{k}\right\}$ defined by the Mann iteration (3.4) with $0 \leqslant x_{1} \leqslant T x_{1}$ and $\mathrm{F}_{\geqslant}^{x_{1}}(T) \neq \emptyset$. If the iteration coefficients $\left\{\alpha_{\mathrm{k}}\right\} \subset(0,1)$ satisfy

$$
\sum_{k=1}^{+\infty} \gamma_{k}=+\infty, \gamma_{k}=\min \left\{\alpha_{k},\left(1-\alpha_{k}\right)\right\} \text { for all positive integers } k,
$$

then $\left\{x_{k}\right\}$ strongly converges to some fixed point $x \in \mathrm{F}_{\geqslant}^{x_{1}}(T)$ and $x \geqslant x_{k}$ for all $k$.

Proof. It follows from Theorem 3.2 that $\left\{x_{k}\right\}$ weakly converges to some fixed point $x \in F_{\geqslant}^{x_{1}}(T)$ and

$$
0 \leqslant x_{1} \leqslant x_{k} \leqslant x_{k+1} \leqslant x \text { for all positive integers } k .
$$

Then from the monotonicity of the norm, it follows that

$$
0 \leqslant\left\|x_{1}\right\| \leqslant\left\|x_{k}\right\| \leqslant\left\|x_{k+1}\right\| \leqslant\|x\| \text { for all positive integers } k,
$$

and hence, the real number sequence $\left\{\left\|x_{k}\right\|\right\}$ is monotone increasing and bounded. Then the limit $\lim _{k \rightarrow \infty}\left\|x_{k}\right\|$ exists and

$$
\lim _{k \rightarrow \infty}\left\|x_{k}\right\| \leqslant\|x\| .
$$

On the other hand, by the weakly lower semi-continuity of the norm, we have

$$
\|x\| \leqslant \liminf _{x \rightarrow \infty}\left\|x_{k}\right\|=\lim _{k \rightarrow \infty}\left\|x_{k}\right\| \leqslant\|x\|,
$$

and so,

$$
\lim _{k \rightarrow \infty}\left\|x_{k}\right\|=\|x\| .
$$

It is well-known that each uniformly convex Banach space has the Kadec-Klee property, i.e.,

$$
\text { weak- } \lim _{n \rightarrow \infty} x_{n}=x \text { and } \lim _{n \rightarrow \infty}\left\|x_{n}\right\|=\|x\| \text { implies } \lim _{n \rightarrow \infty} x_{n}=x \text {. }
$$

Consequently, $\lim _{k \rightarrow \infty} x_{k}=x$. This completes the proof.

Theorem 3.6. Let $\mathrm{K}$ be a nonempty, closed convex subset of a uniformly convex Banach space $\mathrm{E}$ with a partial order " $\leqslant$ ", and let $\mathrm{T}: \mathrm{K} \rightarrow \mathrm{K}$ be a monotone $\alpha$-nonexpansive mapping. Assume that the norm $\|\cdot\|$ is monotonic and the sequence $\left\{x_{k}\right\}$ defined by the Mann iteration (3.4) with $0 \geqslant x_{1} \geqslant T x_{1}$ and $\mathrm{F}_{\leqslant}^{x_{1}}(T) \neq \emptyset$. If the iteration coefficients $\left\{\alpha_{k}\right\} \subset(0,1)$ satisfy

$$
\sum_{k=1}^{+\infty} \gamma_{k}=+\infty, \gamma_{k}=\min \left\{\alpha_{k},\left(1-\alpha_{k}\right)\right\} \text { for all positive integers } k,
$$

then $\left\{\mathrm{x}_{\mathrm{k}}\right\}$ strongly converges to some fixed point $\mathrm{x} \in \mathrm{F}_{\leqslant}^{\mathrm{x}_{1}}(\mathrm{~T})$ and $\mathrm{x} \leqslant \mathrm{x}_{\mathrm{k}}$ for all $\mathrm{k}$.

Proof. It follows from Theorem 3.3 that $\left\{x_{k}\right\}$ weakly converges to some fixed point $x \in F_{\leqslant}^{x_{1}}(T)$ and

$$
0 \geqslant x_{1} \geqslant x_{k} \geqslant x_{k+1} \geqslant x \text { for all positive integers } k \text {. }
$$

Then

$$
0 \leqslant-x_{1} \leqslant-\chi_{k} \leqslant-x_{k+1} \leqslant-x \text { for all positive integers } k .
$$

and hence, from the monotonicity of the norm, it follows that

$$
0 \leqslant\left\|x_{1}\right\| \leqslant\left\|x_{k}\right\| \leqslant\left\|x_{k+1}\right\| \leqslant\|x\| \text { for all positive integers } k .
$$

The remainder of the proof is the same as Theorem 3.5, so we omit it. 
Corollary 3.7. Let $\mathrm{E}$ be a uniformly convex Banach space with the partial order " $\leqslant$ " with respect to the normal cone $\mathrm{P}$, and let $\mathrm{T}: \mathrm{P} \rightarrow \mathrm{P}$ be a monotone $\alpha$-nonexpansive mapping. Assume that the sequence $\left\{\mathrm{x}_{\mathrm{k}}\right\}$ defined by the Mann iteration (3.4) with $\mathrm{x}_{1}=0$ and $\mathrm{F}(\mathrm{T}) \neq \emptyset$. If the iteration coefficients $\left\{\alpha_{\mathrm{k}}\right\}$ satisfy

$$
\sum_{k=1}^{+\infty} \gamma_{k}=+\infty, \gamma_{k}=\min \left\{\alpha_{k},\left(1-\alpha_{k}\right)\right\} \text { for all positive integers } k,
$$

then $\left\{x_{k}\right\}$ strongly converges to some fixed point $x \in F(T)$.

Proof. It is obvious that $F(T)=F_{\geqslant}^{0}(T)=F_{\geqslant}^{x_{1}}(T)$. Since $x_{1}=0$ and $T(P) \subset P$, then

$$
x_{1}=0 \leqslant \mathrm{~T} 0=\mathrm{T} x_{1} .
$$

Then the conclusion directly follows from Theorem 3.5.

\section{Acknowledgment}

This work was supported by the National Natural Science Foundation of P.R. China (Grant No. 11571095, 11361070); The authors are grateful to the editor and anonymous referee for their valuable suggestions which helps to improve this manuscript.

\section{References}

[1] R. P. Agarwal, D. O'Regan, D. R. Sahu, Fixed point theory for Lipschitzian-type mappings with applications, Springer, New York, (2009). 2, 2.5

[2] M. Bachar, M. A Khamsi, On common approximate fixed points of monotone nonexpansive semigroups in Banach spaces, Fixed Point Theory Appl., 2015 (2015), 11 pages. 1, 2.2

[3] V. Berinde, A convergence theorem for Mann iteration in the class of Zamfirescu operators, An. Univ. Vest Timi. Ser. Mat.-Inform., 45 (2007), 33-41. 1

[4] B. A. B. Dehaish, M. A. Khamsi, Mann iteration process for monotone nonexpansive mappings, Fixed Point Theory Appl., 2015 (2015), 7 pages. 1

[5] K. Deimling, Nonlinear Functional Analysis, Dover Publications Inc., New York, (2010). 2

[6] A. B. George, C. A. Nse, A Monotonicity Condition for Strong Convergence of the Mann Iterative Sequence for Demicontractive Maps in Hilbert Spaces, Appl. Math., 5 (2014), 2195-2198. 1

[7] S. George, P. Shaini, Convergence theorems for the class of Zamfirescu operators, Int. Math. Forum, 7 (2012), $1785-1792$. 1

[8] K. Goebel, W. A. Kirk, Topics in metric fixed point theory, Cambridge University Press, Cambridge, (1990). 2

[9] F. Gu, J. Lu, Stability of Mann and Ishikawa iterative processes with errors for a class of nonlinear variational inclusions problem, Math. Commun., 9 (2004), 149-159. 1

[10] J. S. Jung, Iterative approaches to common fixed points of nonexpansive mappings in Banach spaces, J. Math. Anal. Appl., 302 (2005), 509-520. 1

[11] J. K. Kim, Z. Liu, Y. M. Nam, S. A. Chun, Strong convergence theorems and stability problems of Mann and Ishikawa iterative sequences for strictly hemi-contractive mappings, J. Nonlinear Convex Anal., 5 (2004), 285-294. 1

[12] W. A. Kirk, B. Sims, Handbook of metric fixed point theory, Kluwer Academic Publishers, Dordrecht, (2001). 2, 2

[13] F. Kohsaka, W. Takahashi, Existence and approximation of fixed points of firmly nonexpansive-type mappings in Banach spaces, SIAM J. Optim., 19 (2008), 824-835. 1

[14] L.-J. Lin, S.-Y. Wang, Fixed point theorems for ( $\mathrm{a}, \mathrm{b})$-monotone mapping mapping in Hilbert spaces, Fixed Point Theory Appl., 2012 (2012), 14 pages. 1

[15] L. S. Liu, Ishikawa and Mann iteration process with errors for nonlinear strongly accretive mappings in Banach spaces, J. Math. Anal. Appl., 194 (1995), 114-125. 1

[16] W. R. Mann, Mean value methods in iteration, Proc. Amer. Math. Soc., 4 (1953), 506-510. 1

[17] E. Naraghirad, N.-C. Wong, J.-C. Yao, Approximating fixed points of $\alpha$-nonexpansive mappings in uniformly convex Banach spaces and CAT(0) spaces, Fixed Point Theory Appl., 2013 (2013), 20 pages. 1

[18] G. A. Okeke, J. K. Kim, Convergence and summable almost T-stability of the random Picard-Mann hybrid iterative process, J. Inequal. Appl., 2015 (2015), 14 pages. 1

[19] Z. Opial, Weak convergence of the sequence of successive approximations for nonexpansive mappings in Banach spaces, Bull. Amer. Math. Soc., 73 (1967), 591-597. 1

[20] A. Pazy, Asymptotic behavior of contractions in Hilbert spaces, Israel J. Math., 9 (1971), 335-340. 1 
[21] Y. S. Song, R. D. Chen, Pazy's fixed point theorem with respect to the partial order in uniformly convex Banach spaces, preprint arXiv, (2016), 17 pages. 1, 3, 3

[22] Y. S. Song, R. D. Chen, Weak and strong convergence of Mann's-type iterations for a countable family of nonexpansive mappings, J. Korean Math. Soc., 45 (2008), 1393-1404. 1

[23] Y. S. Song, P. Kumam, Y. J. Cho, Fixed point theorems and iterative approximations for monotone nonexpansive mappings in ordered Banach spaces, Fixed Point Theory Appl., 2016 (2016), 11 pages. 1

[24] Y. S. Song, K. Promluang, P. Kumam, Y. J. Cho, Some convergence theorems of the Mann iteration for monotone $\alpha$ nonexpansive mappings, Appl. Math. Comput., 287/288 (2016), 74-82. 1, 2.2, 2.3

[25] Y. S. Song, H. J. Wang, Strong convergence for the modified Mann's iteration of $\lambda$-strict pseudocontraction, Appl. Math. Comput., 237 (2014), 405-410. 1

[26] J. Sun, Nonlinear Functional Analysis and Its Application, Science Publishing House, Beijing, (2008). 2.1

[27] T. Suzuki, Strong convergence of Krasnoselskii and Mann's sequences for one-parameter nonexpansive semigroups without Bochner integrals, J. Math. Anal. Appl., 305 (2005), 227-239. 1

[28] W. Takahashi, Fixed point theorems for new nonlinear mappings in a Hilbert space, J. Nonlinear Convex Anal., 11 (2010), 79-88. 1

[29] W. Takahashi, Nonlinear Functional Analysis-Fixed Point Theory and its Applications, Yokohama Publishers inc, Yokohama, (2000). 2

[30] W. Takahashi, J. C. Yao, Fixed point theorems and ergodic theorems for nonlinear mappings in a Hilbert space, Taiwan. J. Math., 15 (2011), 457-472. 1

[31] H. Zhang, Y. Su, Convergence theorems for strict pseudo-contractions in q-uniformly smooth Banach spaces, Nonlinear Anal., 71 (2009), 4572-4580. 1

[32] H. Y. Zhou, Convergence theorems for $\lambda$-strict pseudo-contractions in 2-uniformly smooth Banach spaces, Nonlinear Anal., 69 (2008), 3160-3173. 1

[33] H. Y. Zhou, M. H. Zhang, H. Y. Zhou, A convergence theorem on Mann iteration for strictly pseudo-contraction mappings in Hilbert spaces (Chinese), J. Hebei Univ. Nat. Sci., 26 (2006), 348-349. 1 